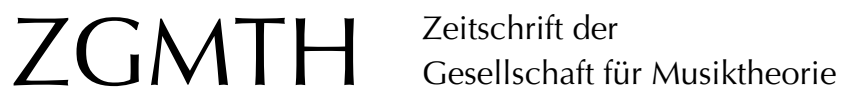

Sprick, Jan Philipp (2015): Felix Wörner / Ullrich Scheideler / Philip Rupprecht (Hg.), Tonality 1900 - 1950. Concept and Practice, Stuttgart: Steiner 2012. ZGMTH 12/1, 147-150. https://doi.org/10.31751/838

(C) 2015 Jan Philipp Sprick

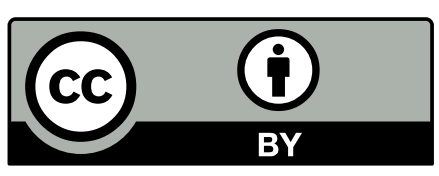

Dieser Text erscheint im Open Access und ist lizenziert unter einer Creative Commons Namensnennung 4.0 International Lizenz.

This is an open access article licensed under a Creative Commons Attribution 4.0 International License.

veröffentlicht / first published: 23/07/2016

zuletzt geändert / last updated: 18/03/2017 


\section{Felix Wörner / Ullrich Scheideler / Philip Rupprecht (Hg.), Tonality 1900-1950. Concept and Practice, Stuttgart: Steiner 2012}

Tonalität ist ein Thema, das im musiktheoretischen Diskurs der vergangenen Jahre verstärkt vertreten ist, wobei sich die Diskussion im deutschsprachigen Raum von derjenigen in der US-amerikanischen Musiktheorie in vielerlei Hinsichten unterscheidet. Vor diesem Hintergrund darf das Anliegen der Herausgeber Felix Wörner, Ullrich Scheideler und Philip Rupprecht als Versuch verstanden werden, die Diskussionsstränge diesseits und jenseits des Atlantiks zusammenzuführen und das Thema `Tonalität - mit dem zusätzlichen Fokus auf die Entwicklung im 20. Jahrhundert - multiperspektivisch zu beleuchten. Der 2012 erschienene Band Tonality 1900-1950. Concept and Practice ist aus einer Konferenz hervorgegangen, die im Jahr 2010 an der University of North Carolina at Chapel Hill und der Duke University stattgefunden hat. Ein zweiter Band, der der Tonalität in der zweiten Hälfte des 20. Jahrhunderts gewidmet ist und dem eine Konferenz in Basel vorausging, ist derzeit in Vorbereitung.

Konzeptioneller Ausgangspunkt des Bandes bildet die Auffassung, nach der Tonalität am Beginn des 20. Jahrhunderts genau zu jener Zeit in den Mittelpunkt der musiktheoretischen Aufmerksamkeit rückte, als die musikalische Avantgarde mehrheitlich ihr Ende proklamierte. ${ }^{1}$ Unter Verweis auf übliche musikhistorische Narrative konstatieren die Herausgeber, dass insbesondere für die Zeit um 1910 gemeinhin die Momente des revolutionären Wandels und nicht des sanften Übergangs betont würden. Beispiele hierfür wären Positionen der Zweiten Wiener Schule, wie diejenige Anton Weberns, der beispielsweise den »Tod" der Tonalität verkündet hatte. Um ihre anderslautende Position von Anfang an deutlich zu machen, scheuen die Herausgeber - wie sie gleich im zweiten Satz der Einleitung

1 Wörner/Scheideler/Rupprecht 2012, 12. einräumen - auch vor einer durchaus polemischen Rhetorik nicht zurück. Wenn in diesem Zusammenhang allerdings suggeriert wird, auf der im Jahr 2009 in Berlin durchgeführten Tagung 100 Jahre Atonalität: Herausforderung für die Musiktheorie sei der vermeintliche ,Geburtstag، der Atonalität unreflektiert gefeiert worden, so verdeutlicht dies allenfalls die generelle Tendenz des Bandes, sich ostentativ quer zu etablierten Diskursen und Narrativen zu stellen; denn auch die Berliner Tagung hatte ihren Anlass primär in der Problematisierung gängiger Erzählungen über Tonalität und Atonalität und nicht in deren Affirmation. ${ }^{2}$

Die Geschichte, die Herausgeber und Autorinnen und Autoren im zu besprechenden Band erzählen, betont vor allem die Kontinuität tonaler Musik für den Zeitraum von 1900-1950, repräsentiert durch Komponisten wie Sibelius, Debussy, Copland, Prokofiev, Poulenc, Tippett und viele andere. Daneben bildet die Aufarbeitung der theoretischen Reflexion über Tonalität einen weiteren Schwerpunkt des Bandes, die - den Entwicklungen in der musikalischen Praxis mitunter entgegengesetzt - gerade zu Beginn des 20. Jahrhunderts eine Konjunktur erlebt hatte, was sich insbesondere in den Schriften von Schönberg, Schenker, Kurth und Riemann widerspiegelt.

Der verdienstvolle Ausgangspunkt und die zentrale Fragestellung des Bandes ist, wie man der Vielfalt tonalen Komponierens in der ersten Hälfte des 20. Jahrhunderts analytisch gerecht werden kann. Wer allerdings erwartet, dass sich verallgemeinerbare kompositorische Strategien herausschälen, wird zwangsläufig enttäuscht. Für die Herausgeber gibt es zwar einige verbindende Elemente im tonalen Komponieren in der ersten Hälfte des 20. Jahrhunderts, doch sehen sie sich

2 Die Beiträge des Symposions sind in Hohmaier 2009 erschienen. 
gezwungen zu konstatieren, dass Tonalität nach 1900 der sprachlichen Vertrautheit und Sicherheit früherer Zeiten entbehre (»tonality after 1900 lacks the kind of linguistic familiarity and security observable in music of earlier periods", 14). Insofern scheint es nur konsequent, dass die Herausgeber bereits am Ende der Einleitung den weitergehenden Forschungsbedarf auf diesem Gebiet feststellen.

Der Band gliedert die Texte in drei Gruppen. Den Anfang machen vier Beiträge unter der Überschrift »Tonality as Concept and Category«. Joseph Auner zeigt in "Weighting, Measuring, Embalming Tonality: How we Became Phonometrographers " materialreich den Zusammenhang zwischen kompositorischen und technischen Entwicklungen in der ersten Hälfte des 20. Jahrhunderts auf. Die Position dieses Beitrags ganz zu Beginn des Bandes steht für den Anspruch, das Thema ıTonalitätı nicht nur musikimmanent, sondern auch kulturell und technologisch zu kontextualisieren, der dann freilich nicht von allen folgenden Beiträgen eingelöst werden kann. Auch der Beitrag von Richard Cohn findet sich unter Verweis auf die Rolle des Hörers bei der Identifizierung tonaler Effekte hier eingruppiert, obgleich er sich als interessante und detaillierte Analyse von Prokofievs Peter und der Wolf entpuppt und insofern auch unter den analytischen Fallstudien des dritten Teils hätte abgedruckt werden können. In kenntnisreichen Beiträgen geben Wolfgang Rathert und Hans-Joachim Hinrichsen einen differenzierten Überblick über die Rezeption der "German Rule« - also deutschsprachiger Musiktheorie - in den USA, sowie eine subtile Beschreibung der Wandlungen in Hindemiths Tonalitätsbegriff. Insbesondere die Platzierung des letztgenannten Beitrags macht auf gewisse Inkonsistenzen bei der Gliederung des Bandes aufmerksam: Sein Titel - »Concepts of Tonality in Hindemith's Unterweisung im Tonsatz and in his late writings " - ist bis auf den Untersuchungsgegenstand identisch mit demjenigen des Beitrags von Markus Böggemann »Concepts of Tonality in Schoenberg's Harmonielehre", der die zweite Artikelgruppe unter der Überschrift »Tonality in Austro-Ger- man Theory« eröffnet. In diesem Zusammenhang fällt auf, dass ein grundlegender, überblicksartiger Beitrag zur Frage, was Tonalität überhaupt konstituiert und welche Antworten hierauf historisch und aktuell gegeben werden, im ersten Teil des Bandes fehlt. Er hätte es unter Umständen ermöglicht, für den angestrebten transatlantischen Dialog einen gemeinsamen Bezugspunkt zu schaffen. So bleibt es hier bei der zu Beginn von den Herausgebern gegebenen Definition, Tonalität werde im vorliegenden Band als "the awareness of key in music (11) verstanden.

In der zweiten Artikelgruppe stehen zwei Beiträge zu Arnold Schönbergs Harmonielehre neben einem Beitrag zur Energetik Ernst Kurths. Markus Böggemann wendet sich zwei konkurrierenden Konzepten von Tonalität in Schönbergs Harmonielehre zu: Tonalität als natürliches und als historisches Phänomen. Stephen Hinton stellt in seinem Beitrag "Schoenberg's Harmonielehre: Psychology and Comprehensibility", eine vergleichende Untersuchung zu den drei Fassungen der Harmonielehre zwischen 1910 und 1922 an und wirft die Frage auf, inwiefern die Harmonielehre als eine Theorie bezeichnet werden kann. Böggemann, der in seinen Ausführungen nur die erste Auflage heranzieht, betont demgegenüber, dass Schönberg je nach argumentativer Notwendigkeit unterschiedliche Konzepte von Tonalität aufruft. Stephen Hinton schließlich erkennt einen Wandel, den er im Hinblick auf die Begriffe »Emanzipation der Dissonanz« und »Fasslichkeit« diskutiert und mit der Entwicklung von Schönbergs kompositorischer Ästhetik abgleicht. Hintons erfolgreicher Versuch, Geschichte der Musiktheorie und Kompositionsgeschichte ins Gespräch zu bringen, fungiert als Bindeglied zwischen den theoretisch-historischen Beiträgen und den analytischen Fallstudien. Den Abschluss der theoretisch-historischen Beiträge setzt Felix Wörners präzise argumentierende Rekonstruktion von Ernst Kurths Tonalitätskonzept, das nur unter Einbeziehung zeitgenössischer philosophischer Konzepte - wie denjenigen von Dilthey oder Bergson - überhaupt zu verstehen sei. 
Die größte Gruppe des Bandes bilden dann acht Fallstudien des abschließenden dritten Teils, die unter der Überschrift "Practices of Tonality « unterschiedliche Repertoires bearbeiten, die sich zwischen Deutschland, Frankreich, Großbritannien und den USA bewegen. Die geographische Auswahl der Beispiele kann dabei als eine erste Antwort auf die Frage nach Gründen für die in der Einleitung des Bandes konstatierte einseitige Geschichtsbetrachtung hinsichtlich der sTonalitätsgrenze 1910 verstanden werden: Während die in dem Band thematisierten Theoretiker fast ausnahmslos aus dem deutsch-österreichischen Bereich kommen, ist die zu dieser Zeit komponierte Musik aus diesen Ländern in den Fallstudien so gut wie nicht präsent.

Die ersten drei Einzeluntersuchungen sind französischer Musik gewidmet. Marianne Wheeldon kontextualisiert in ihrem Beitrag »Defending Tonality: The Musical Thought of Milhaud and Koechlin « die in den 1920er Jahren in Frankreich breit diskutierten Begriffe Polytonalität und Atonalität und stellt diese dem zeitgenössischen Tonalitätsverständnis gegenüber. Wheeldons Ausführungen verdeutlichen, dass diese Begriffe keineswegs nur Gegenstand hochspezialisierter musiktheoretischer Fachdiskurse waren, sondern auch in ästhetischen Debatten eine wichtige Rolle spielten. Die Protagonisten Milhaud und Koechlin verbinden Wheeldons Beitrag mit Mark Delaeres Text »Polytonality in French Music Theory and Composition of the 1920s". Delaere, bei dem die musiktheoretische Perspektive eine wichtigere Rolle einnimmt als bei Wheeldon, gelingt eine detaillierte Darstellung der Entwicklungsgeschichte des Begriffs "Polytonality" innerhalb der französischen Musiktheorie der 1920er Jahre. Der Vergleich mit zeitgenössischen Kompositionen realisiert eine Gegenüberstellung von Musiktheorie und kompositorischer Wirklichkeit, die den Untertitel des Bandes - „Concept and Practice« - in überzeugender Weise umsetzt. Volker Helbing steuert zur Diskussion französischer Musik eine äußerst genaue und originelle Analyse von Ravels Sonate pour violon et violoncelle bei, die in erster Linie der tona- len und formalen Dramaturgie gewidmet ist. Helbings Analyse ist im besten Sinne eigenständig, indem sie ihre konkrete Fragestellung unmittelbar an der musikalischen Struktur entwickelt. Der wesentliche Ertrag des Artikels besteht in den subtilen analytischen Erkenntnissen, weniger in der Kontextualisierung des Stücks, die in einer Reihe von Beiträgen des Bandes die analytischen Resultate zu überdecken scheinen. Ein Beleg für die nach wie vor relativ unabhängig voneinander laufenden Diskurse in den USA und Deutschland ist die Tatsache, dass Helbing in einer längeren Fußnote im Zusammenhang mit Kleinterzachsen auf einer Mittelgrund-Ebene explizit auf die in Deutschland mittlerweile breit rezipierte Tonfeld-Theorie des ungarischen Dirigenten und Musiktheoretikers Albert Simon verweist und damit auf einen Diskurs, der auch bereits im Jahr 2010 eine gewisse Konjunktur hatte, die es hätte sinnvoll erscheinen lassen, einen Grundlagenbeitrag in den ersten Teil des Bandes mitaufzunehmen. Zudem wären dadurch interessante Überschneidungspunkte mit der Neo-Riemannian Theory deutlich geworden, die im Mittelpunkt des Beitrags von Richard Cohn steht. Verbindet man hierzulande Riemanns Denken zumeist mit einer inzwischen historischen Form der Funktionstheorie, bildet es in der anglo-amerikanischen Musiktheorie einen aktuellen Ausgangspunkt der Theorieentwicklung. Auf diese Weise wäre dem durch den vorliegenden Band vermittelten Eindruck entgegengewirkt worden, wonach die US-amerikanische Musiktheorie die Entwicklung neuer theoretischer Systeme vorantreibt, während die deutschsprachige Musiktheorie überwiegend von einer historisch-kontextualisierenden, gerade nicht systematisch arbeitenden Musikwissenschaft dominiert werde. Dieser Eindruck wird durch die jeweilige institutionell-disziplinäre Herkunft der Autorinnen und Autoren noch verstärkt. Auch wenn das Bemühen erkennbar ist, neben dem transatlantischen Dialog auch einen Dialog zwischen historischer Musikwissenschaft und Musiktheorie herzustellen, bilden aus dem deutschsprachigen Bereich Vertreter der historischen Musikwissenschaft die Mehr- 
heit, während die US-amerikanische Musikforschung überwiegend durch die akademische Musiktheorie repräsentiert wird. Gerade die Vertreter der Tonfeld-Theorie haben den Übergang zwischen unterschiedlichen `Tonalitätsprogrammen thematisiert und alternative Sichtweisen auf die herkömmliche Dichotomie Tonalität versus Atonalität angeboten.

Die Fallstudien vier bis sechs stellen mit einer Studie zur Tonalität in Vaughan Williams A London Symphony, der sich Alain Frogley mit einer hermeneutischen Lesart nähert, und Philip Rupprechts Beitrag zu Brittens »Triadic Modernism, 1930-1940« zweimal die englische Musik in den Mittelpunkt. Rupprecht zeichnet mit seinen überzeugenden und im Hinblick auf Brittens Werk breit kontextualisierten Analysen, insbesondere des Sextet for Wind (1930) und „Villes» (1939), ein Bild des englischen Komponisten, das bewusst eine andere Art des musikalischen Fortschritts in den Fokus rückt, als diejenige der kontinentalen Avantgarde. Die Tatsache, dass auch Rupprecht bei der Analyse auf die Neo-Riemannian Theory zurückgreift weist den Artikel eindeutig als dem anglo-amerikanischen Diskurs zugehörig aus, in dem auch Einzelfallbetrachtungen zumeist theoriegeleitet sind. Ullrich Scheideler widmet sich mit der Untersuchung der Tonalität in Amateurmusik aus Deutschland um 1930 einem originellen Thema und demonstriert an Werken von Bruno Stürmer, Kurt Weill und Paul Hindemith die großen Unterschiede, die tonales Komponieren in dieser Zeit kennzeichnen. Mit Roy Harris und Samuel Barber stehen in den Beiträgen von Beth E. Levy und Daniel Harrison abschließend noch zwei US-Komponisten im Fokus, deren teilweise affirmatives Verhältnis zu tonalem Komponieren ein Gegenmodell zu den europäischen Avantgarde-Diskursen repräsentiert.

Insbesondere die Beiträge in den ersten beiden Sektionen liefern jeder für sich sehr interessante Einsichten, machen aber in ihrer Zusammenstellung auf die brüchige Gliederung des Bandes aufmerksam. Gerade die auf die Geschichte der Musiktheorie fokussierten Beiträge sind weniger auf Theorien gerichtet, die die tonale Praxis der ersten Jahrhunderthälfte in den Blick nehmen, als vielmehr auf Tonalitätstheorien, die sich in der ersten Hälfte des 20. Jahrhunderts mit der Frage der Tonalität beschäftigen. So ist es denn letztlich die Aufarbeitung der musiktheoretischen Diskurse, die in dem Band eine größere Rolle spielt als es die Einleitung zunächst suggeriert. Auffällig ist insbesondere, dass die fünf Beiträge, die dezidiert der Geschichte der Musiktheorie gewidmet sind, sich überwiegend mit Musiktheorie beschäftigen, die die Analyse historisch älterer Musik zum Gegenstand hat und ausdrücklich nicht die Musik der ersten Hälfte des 20. Jahrhunderts. Fraglos ist es relevant, historische Tonalitätstheorien dezidiert in den Blick zu nehmen, nur hätte hier - soll das zentrale Interesse auf der analytischen Beschäftigung mit dem tonalen Repertoire liegen - ein stärkerer Zusammenhang zwischen Theorie und zeitgenössischer Musik hergestellt werden müssen.

Gleichwohl bietet der sehr sorgfältig produzierte und lektorierte Band eine beeindruckende Vielfalt an Perspektiven auf einen hochaktuellen Gegenstand und wendet sich einem großen Desiderat in der derzeitigen Publikationslandschaft zu. Auf den in Kürze erscheinenden zweiten Band über die zweite Hälfte des 20. Jahrhunderts darf man gespannt sein.

Jan Philipp Sprick

\section{Literatur}

Hohmaier, Simone (Hg.) (2009), Jahrbuch 2008/09 des Staatlichen Instituts für Musikforschung Preußischer Kulturbesitz, Mainz: Schott. 